\title{
Influence of Thiazole-modified Carbon Nitride Nanosheets with Feasible Electronic Properties on Inverted Perovskite Solar Cells
}

Daniel Cruz, ${ }^{\mathrm{a}, \ddagger}$ Jose Garcia Cerrillo,, ,ł Baris Kumru, ${ }^{\mathrm{a}}$ Ning Li, b,,e Jose Dario Perea, ${ }^{\mathrm{b}, \mathrm{f}}$ Bernhard V.K.J. Schmidt, ${ }^{\mathrm{a}}$ Iver Lauermann, ${ }^{\mathrm{d}}$ Christoph J. Brabec, ${ }^{\mathrm{b}, \mathrm{c}, \mathrm{g} *}$ and Markus Antonietti ${ }^{\mathrm{a}, *}$

a. Max-Planck-Institute of Colloids and Interfaces; Department of Colloid Chemistry, Am Mühlenberg 1, 14476 Potsdam, Germany

b. Institute of Materials for Electronics and Energy Technology (i-MEET), Friedrich-Alexander University Erlangen-Nürnberg, Martensstraße 7, 91058 Erlangen, Germany

c. Helmholtz-Institute Erlangen-Nürnberg for Renewable Energy, Immerwahrstraße (HI-ErN) 2, 91058 Erlangen, Germany

d. Kompetenzzentrum Dünnschicht- und Nanotechnologie für Photovoltaik Berlin (PVcomB), Helmholtz-Zentrum Berlin für Materialien und Energie GmbH,-Schwarzschildstraße 3,D-12489 Berlin Germany

e. National Engineering Research Center for Advanced Polymer Processing Technology, Zhengzhou University, Zhengzhou, 4500o2, China

f.Photovoltaic Research Laboratory, Massachusetts Institute of Technology (MIT), Cambridge, MA 02139 USA

g. Forschungszentrum Jülich GmbH (FZJ), Wilhelm-Johnen straße, 52428 Jülich, Germany

\section{Experimental Section}

Materials: Barbituric acid (99\%, Alfa Aesar), cyanuric acid (98\%, Sigma Aldrich), melamine (99\%, Sigma Aldrich) 4-methyl-5-vinyl thiazole (vTA, 97\%, Kosher, Sigma Aldrich). Poly[bis(4-phenyl)(2,4,6-trimethylphenyl)amine] (PTAA, Sigma Aldrich, average $M_{\mathrm{n}}$ of 7,00010,000), lead(II) iodide (TCI Chemicals), methylammonium iodide (Lumtec), N-Methyl-2pyrrolidone (NMP, Sigma Aldrich, 99.5\%), 2-propanol (IPA Sigma Aldrich, $\geq 99.5 \%$ ), acetonitrile (ACN Sigma Aldrich, $\geq 99.5 \%$ ), dimethylformamide (DMF, Sigma Aldrich, $\geq 99 \%$ ), [6,6]-phenyl- $\mathrm{C}_{61}$-butyric acid methyl ester (PCBM, Solenne, 99.5\%), and aluminum-doped zinc oxide (AZO, Avantama) ink from Nanograde (product no. N-20X). were used to fabricate solar cell devices. 50W LED chips (Foxpic High Power 50 W LED Chip Bulb Light DIY White 3800LM $6500 \mathrm{~K}$ ) were connected to a self-made circuit and cooling system. 
Preparation of CMB: $1.0 \mathrm{~g}$ of cyanuric acid (C), $0.1 \mathrm{~g}$ barbituric acid (B) and $1.0 \mathrm{~g}$ of melamine (M) were mixed with $40 \mathrm{~mL}$ distilled water and shaked overnight. After centrifugation at $5000 \mathrm{rpm}$ for 5 minutes. The precipitate was dried at $60{ }^{\circ} \mathrm{C}$ under vacuum overnight. The dried product was transferred into a capped crucible and put into $\mathrm{N}_{2}$ protected oven at $550{ }^{\circ} \mathrm{C}$ for 4 hours, with a heating rate of $2.3{ }^{\circ} \mathrm{C} / \mathrm{min} .{ }^{1}$

Modification of CMB with vTA: $300 \mathrm{mg} \mathrm{CMB}$ was mixed with $2 \mathrm{~mL}$ vTA and sonicated for 5 minutes in a sonication bath. The mixture was placed between $250 \mathrm{~W}$ LED set ups and stirred for 4 hours under continuous visible light irradiation. For purification, mixture was filtered and washed with ethanol 3 times ( $20 \mathrm{~mL}$ each portion) and dried under vacuum at $60{ }^{\circ} \mathrm{C}$ overnight. ${ }^{2}$

Device fabrication: Laser-patterned, ITO-coated glass substrates were cleaned by sequential ultrasonication for $10 \mathrm{~min}$ in acetone and isopropanol, respectively. Thereafter, the substrates were subjected to an $\mathrm{O}_{2}$-plasma cleaning procedure for $2 \mathrm{~min}$ to ensure further removal of organic residues on the ITO surface.

PTAA was dissolved in chlorobenzene (Acros) at a concentration of $1 \mathrm{mg} / \mathrm{mL}$. Lead(II) iodide and methylammonium iodide were added to a mixture of $20 \%$ of NMP and DMF at a molar ratio of 1:1 to prepare a precursor ink with a concentration of 1.60 M. PCBM was dissolved in chlorobenzene at a concentration of $20 \mathrm{mg} / \mathrm{mL}$, and Al-doped Zin oxide( AZO) ink from Nanograde (product no. N-20X).

The PTAA precursor solution was spin coated on the cleaned ITO substrates at a spin speed of $4000 \mathrm{rpm}$ for $20 \mathrm{~s}$, and then annealed at $110{ }^{\circ} \mathrm{C}$ for $10 \mathrm{~min}$ inside a $\mathrm{N}_{2}$ glovebox.

The $\mathrm{MAPbI}_{3}$ precursor ink was subsequently spin coated on the DMF pre-wetted substrates in two steps: $3000 \mathrm{rpm}$ for $25 \mathrm{~s}$ and $6000 \mathrm{rpm}$ for 35 s. $200 \mu \mathrm{L}$ of chlorobenzene was added on top of the spinning substrate $7 \mathrm{~s}$ prior to the end of the first step. After wards, CMB-vTA, PCBM and AZO suspensions were deposited separately on top of the perovskite film at a spin speed of $2000 \mathrm{rpm}$ for $30 \mathrm{~s}$ and $10 \mathrm{~s}$, and immediately annealed after deposition at $110^{\circ} \mathrm{C}$ for $1 \mathrm{~min}$ and $5 \mathrm{~min}$, respectively. Finally, $100 \mathrm{~nm}$ of silver were evaporated on top of the stack using a patterned mask to define an active area of $0.104 \mathrm{~cm}^{2}$.

\section{Characterization of CMB-vTA}

Particle charges of colloidal dispersed g-CNs were detected with Mütek PCD 03 particle charge detector. X-ray diffraction (XRD) patterns were obtained using Bruker D8 Advance X-ray 
diffractometer via $\mathrm{Cu}-\mathrm{K}_{\alpha}$ radiation. High resolution transmission electron microscopy (HRTEM) measurements were acquired using a double-corrected Jeol ARM200F, equipped with a cold field emission gun and a Gatan GIF Quantum. The used acceleration voltage was $200 \mathrm{kV}$ and the emission was set to $10 \mu \mathrm{A}$ in order to reduce beam damage. Combustive elemental analysis of g-CN was recorded via a Vario Micro device. Solid state ultraviolet-visible (UVVis) spectroscopy was recorded via a Cary 500 Scan spectrophotometer equipped with an integrating sphere. Photoluminescent emission spectra were recorded on Jasco FP-8300 instrument with the excitation wavelength at $360 \mathrm{~nm}$.

\section{TRPL spectroscopy distribution of fluorescence lifetimes}

Time resolve photoluminescence study was performed by exciting $405 \mathrm{~nm}$ wavelength using a pulse width $4 \mu \mathrm{s}$ through $450 \mathrm{~nm}$ filter $\mathrm{o}$ and the emission dispersed with an $\mathrm{H} 10$ monochromator, and dectected using a 9816 photomultiplier (EMI) with digital oscilloscope TDS540(Tektronix) . To determine lifetimes were used an iterative reconvolution that described the behave for a double-exponential decay follow model:

$$
I(t)=\sum_{m} A_{m} e^{-\frac{t}{\tau_{m}}}
$$

Where $\mathbf{I}(\mathbf{t})$ is the observaded intensity, $\tau_{m}$ lifetime is a compomnent of the succession $\mathrm{m}$, and $\mathrm{A}_{\mathrm{m}}$ is a preexponential term relate to amplitud.

Now, in order to calculate the average flourescence lifetime is used the following relation:

$$
\langle\tau\rangle=\frac{\sum_{i=1}^{2} a_{i} \tau_{i}^{2}}{\sum_{i=1}^{2} a_{i} \tau_{i}}
$$

\section{Device Characterization}

The characterization of the solar cells was performed via a Keithley 7001 source measurement unit coupled with an Oriel Newport Sol1A solar simulator with an AM1.5G spectrum at 100 $\mathrm{mW} / \mathrm{cm}^{2}$ (previously calibrated with a $\mathrm{Si}$ solar cell as a reference) to characterize the photovoltaic performance of the complete, masked solar cells. The measurements were performed by applying a forward bias at a voltage sweep of $50 \mathrm{mV} / \mathrm{s}$. 
The external quantum efficiency (EQE) of the complete devices was measured with an Enli Technology measurement unit. A silicon cell was used as a previous reference to measure the experimental devices. The steady-state and time-resolved photoluminescence spectra of perovskite films deposited on glass and/or ITO-coated glass were obtained with a Fluorescence Lifetime Spectrometer PicoQuant FluoTime 300 equipped with a PicoQuant Computer Controlled Picosecond Diode Laser PDL 820 of $402 \mathrm{~nm}$. The contact angle profile of CMB-TA on perovskite MAPI thin film were determined by droplet shape profile analysis from OCA instrument, data physics ES, Germany, and drop shape analysis dsa1 V1.80 Krüss software.

\section{Ultraviolet Photoelectron Spectroscopy (UPS) features:}

UPS measurements were carried out with CISSY station at Hemholtz Zentrum Berlin, under ultra-high vacuum (UHV) $1.5 \times 10^{-8} \mathrm{~Pa}$ and the reference binding energy scale was calibrate using $\mathrm{Au} 4 \mathrm{f}_{7 / 2}(84.0 \mathrm{eV})$. The station is equipped with of He I $21.2 \mathrm{eV}$ and UVS 10/35and combined with lens analyzer module (CLAM).

\section{Field Emission Scanning Electron Microscope (FE-SEM)}

Top-view images of perovskite films with and without CMB-vTA were taken with a JSM7610F high resolution Field Emission Scanning Electron Microscope at an accelerating voltage of $3.00 \mathrm{kV}$ and magnifications of 50.000 and $75.000 \mathrm{X}$. 
Table S1. Combustive elemental analysis results of non-modified (CMB) and vTA modified (CMB-vTA) carbon nitrides.

\begin{tabular}{|l|c|c|c|c|}
\hline & N (\%) & $\mathbf{C ~ ( \% )}$ & $\mathbf{S ~ ( \% )}$ & $\mathbf{C} / \mathbf{N}$ \\
\hline CMB & 52.11 & 45.88 & 0.342 & 0.88 \\
\hline CMB-vTA & 47.43 & 49.21 & 3.211 & 1.03 \\
\hline
\end{tabular}

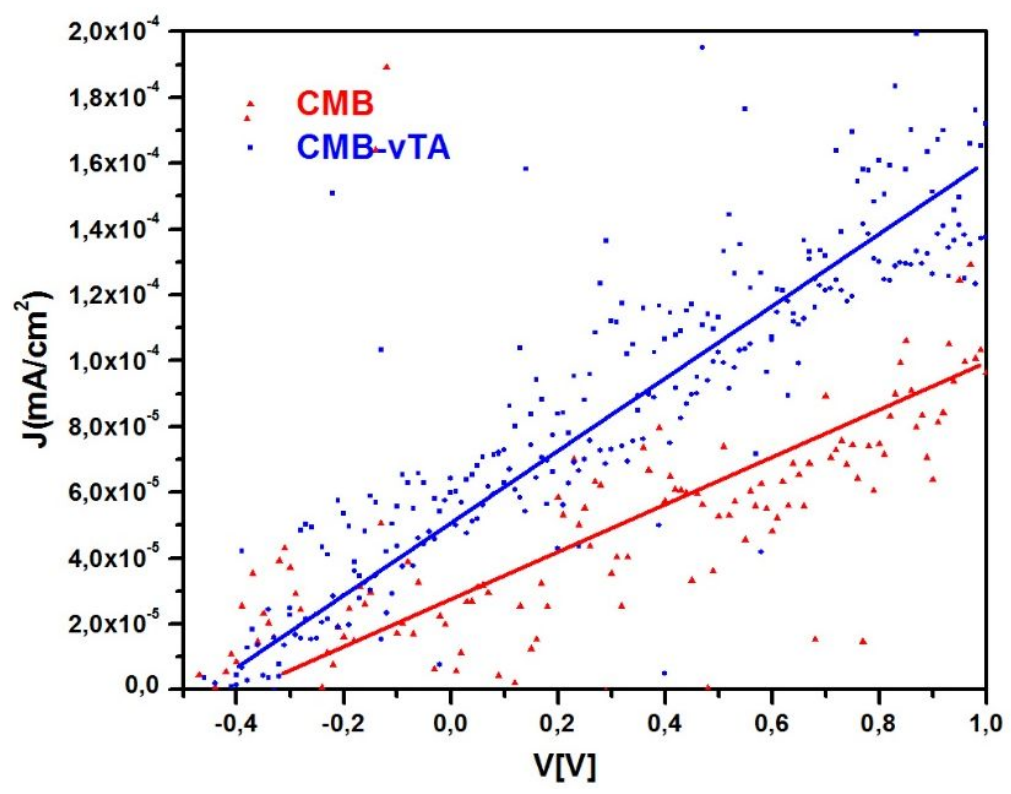

Figure S1. Current density and voltage of CMB and CMB-vTA films.

Table S2. Particle charge for CMB-vTA dispersion in IPA-ACN 1:1

\begin{tabular}{|l|c|}
\hline & $1: 1$ IPA:ACN \\
\hline CMB-vTA & $-103.6 \mathrm{mV}$ \\
\hline
\end{tabular}




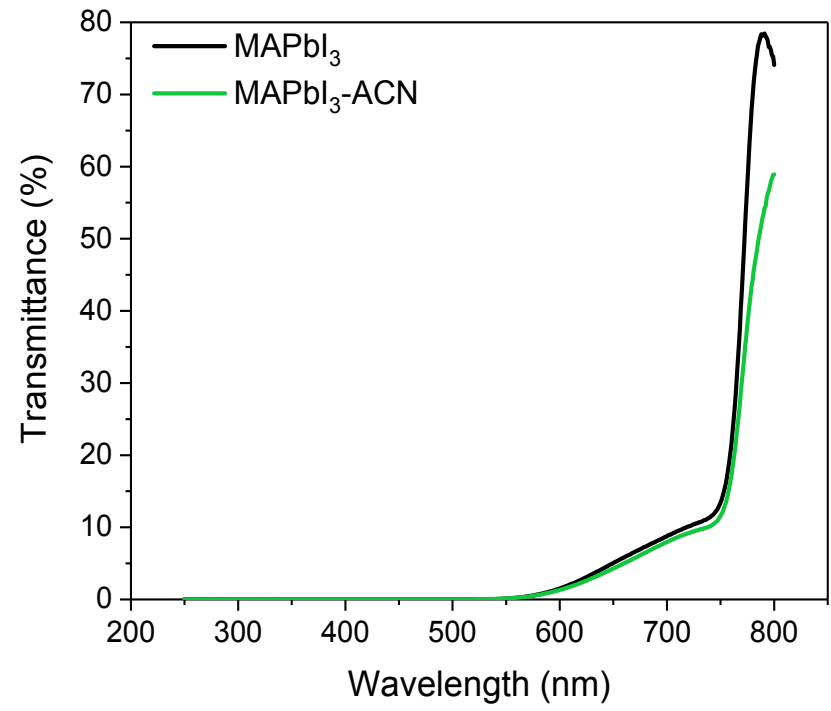

Figure S2. Transmittance measurements for pure $\mathrm{MAPbI}_{3}$ films and films treated with $\mathrm{ACN}$. 

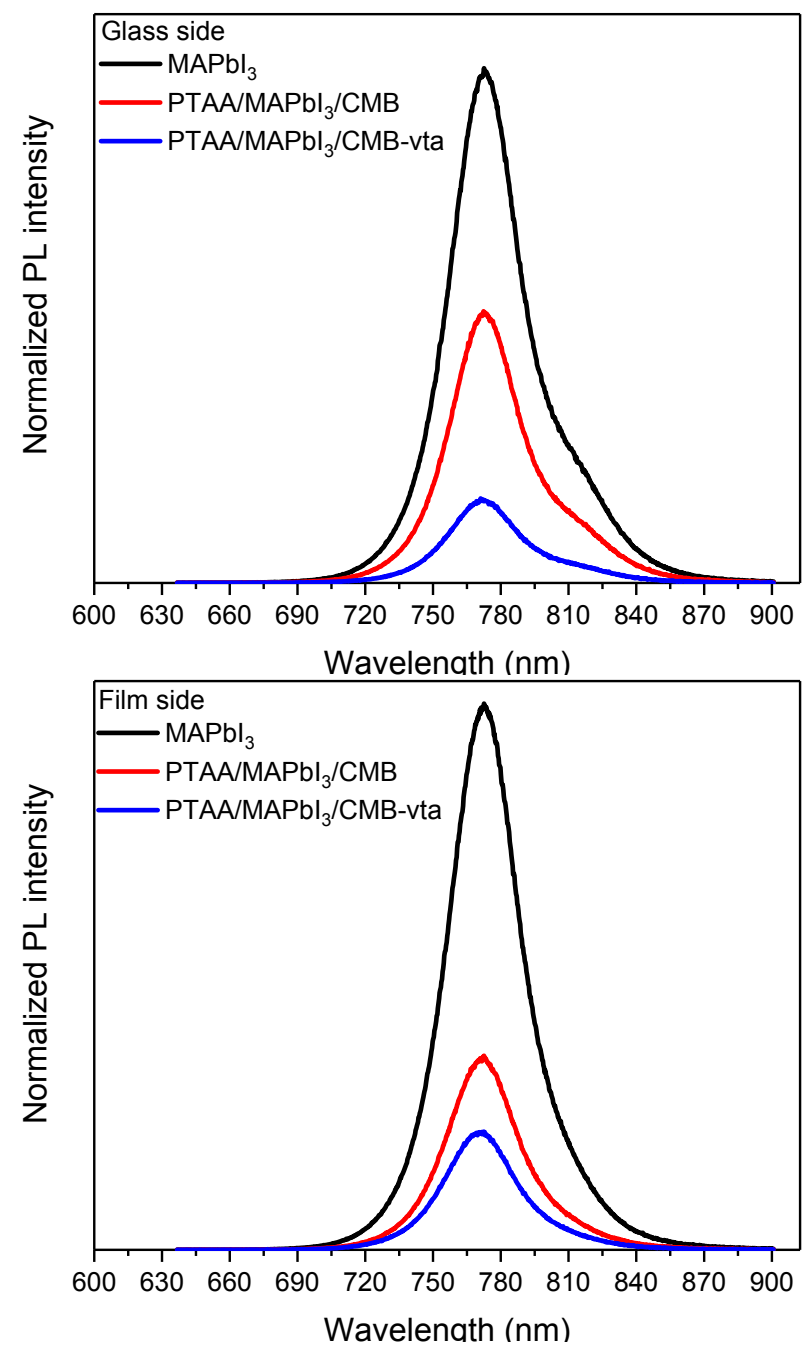

Figure S3. Photoluminescence PL decay of Glass/PTAA/Perovskite with CMB and CMB-vTA by illumination on glass and film side. 
Table S3. TRPL parameters for the structure Glass/PTAA/Perovskite with CMB or CMBvTA

\begin{tabular}{|c|c|c|c|c|c|c|c|}
\hline \multicolumn{8}{|c|}{ Measurements on the glass side } \\
\hline Stack on glass & $\tau_{1}(\mathrm{~ns})$ & $A_{1}$ (cts) & $\tau_{2}(\mathrm{~ns})$ & $A_{2}$ (cts) & Bkgr. dec. & $X^{2}$ & $\tau_{\text {avg }}(\mathrm{ns})$ \\
\hline $\mathrm{MAPbI}_{3}$ & 167.04 & 293.42 & 72.58 & 271.00 & 2.306 & 0.8768 & 139.99 \\
\hline $\mathrm{PTAA} / \mathrm{MAPbI}_{3} / \mathrm{CMB}$ & 69.59 & 249.59 & 24.42 & 183.50 & 0.766 & 0.6307 & 60.33 \\
\hline $\mathrm{PTAA} / \mathrm{MAPbI}_{3} / \mathrm{CMB}$-vta & 3.894 & 405.10 & 18.32 & 369.88 & 0.858 & 0.7215 & 15.59 \\
\hline \multicolumn{8}{|c|}{ Measurements on the film side } \\
\hline Stack on glass & $\tau_{1}(\mathrm{~ns})$ & $A_{1}$ (cts) & $\tau_{2}(\mathrm{~ns})$ & $A_{2}$ (cts) & Bkgr. dec. & $X^{2}$ & $\tau_{\text {avg }}(\mathrm{ns})$ \\
\hline $\mathrm{MAPbI}_{3}$ & 48.01 & 156.30 & 167.72 & 402.00 & 3.129 & 0.8990 & 155.73 \\
\hline $\mathrm{PTAA} / \mathrm{MAPbI}_{3} / \mathrm{CMB}$ & 3.54 & 288.40 & 23.67 & 472.40 & 0.786 & 0.5918 & 21.99 \\
\hline $\mathrm{PTAA} / \mathrm{MAPbI}_{3} / \mathrm{CMB}$-vta & 2.18 & 248.10 & 9.42 & 532.30 & 1.192 & 0.7325 & 8.71 \\
\hline
\end{tabular}

a)

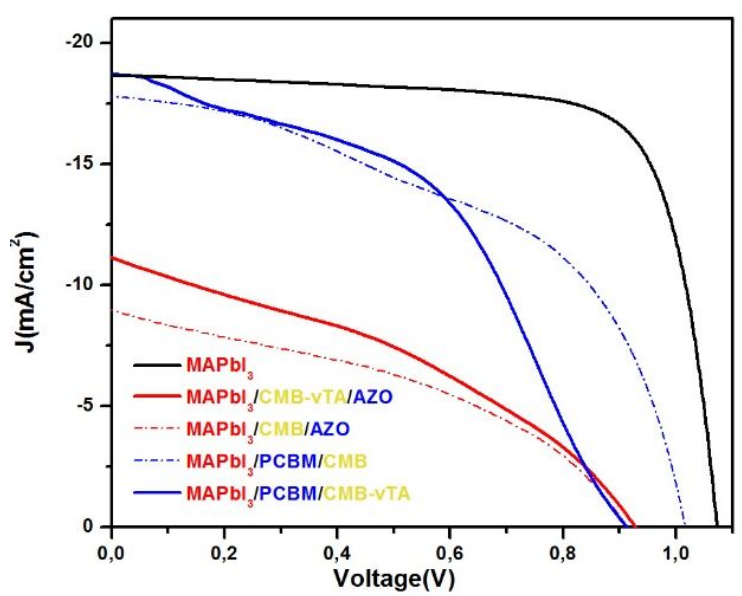

b)

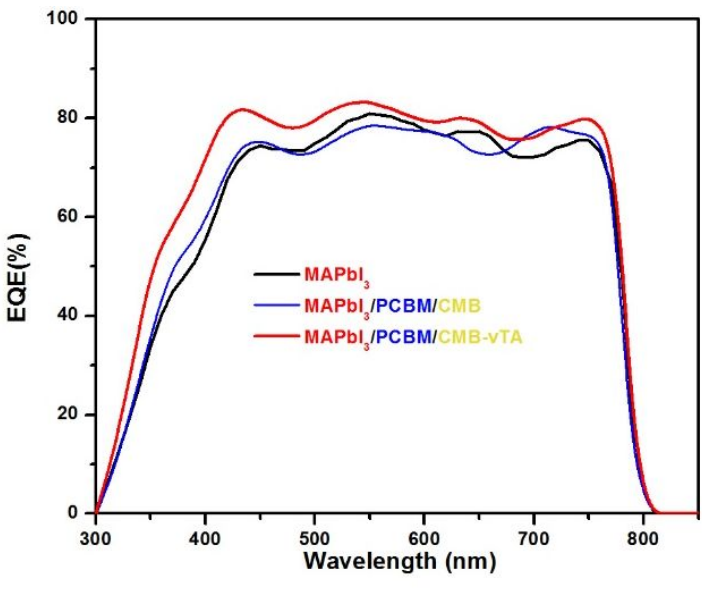

Figure S4. a) Current density versus voltage characteristics of $0.104 \mathrm{~cm}^{2} \mathrm{MAPbI}_{3}$ based solar cells with and without CMB-vTA, b) external quantum efficiency spectra of $\mathrm{MAPbI}_{3}$ solar cells with and without CMB-vTA. 


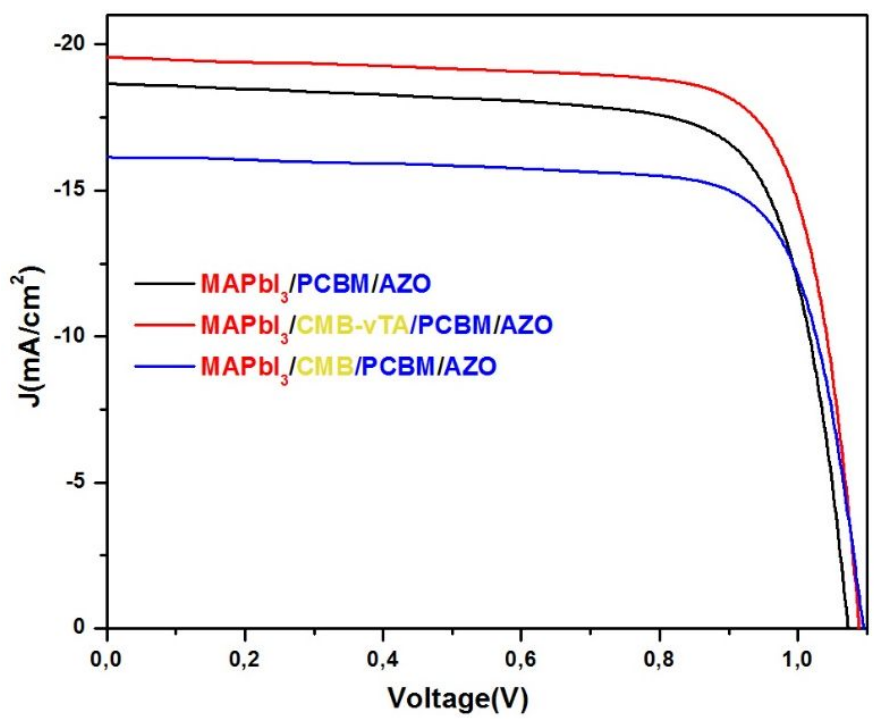

Figure S5. Current density versus voltage characteristics of $0.104 \mathrm{~cm}^{2} \mathrm{MAPbI}_{3}$ based solar cells with and without CMB-vTA located at the interface. 

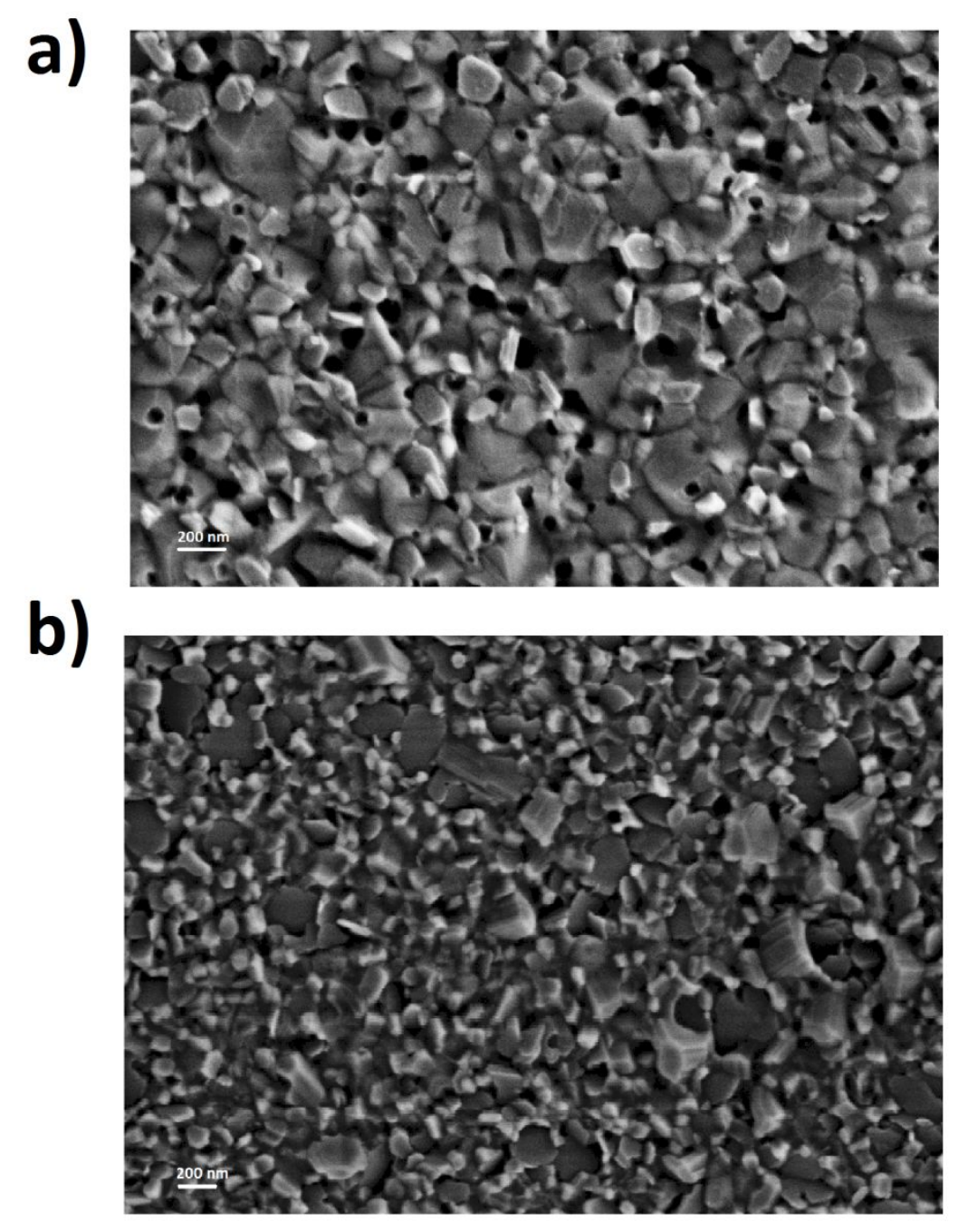

Figure S6. SEM images for thin films of a) methyl ammonium lead iodide (MAPI) and b) CMB-vTA deposited on methyl ammonium lead iodine. 


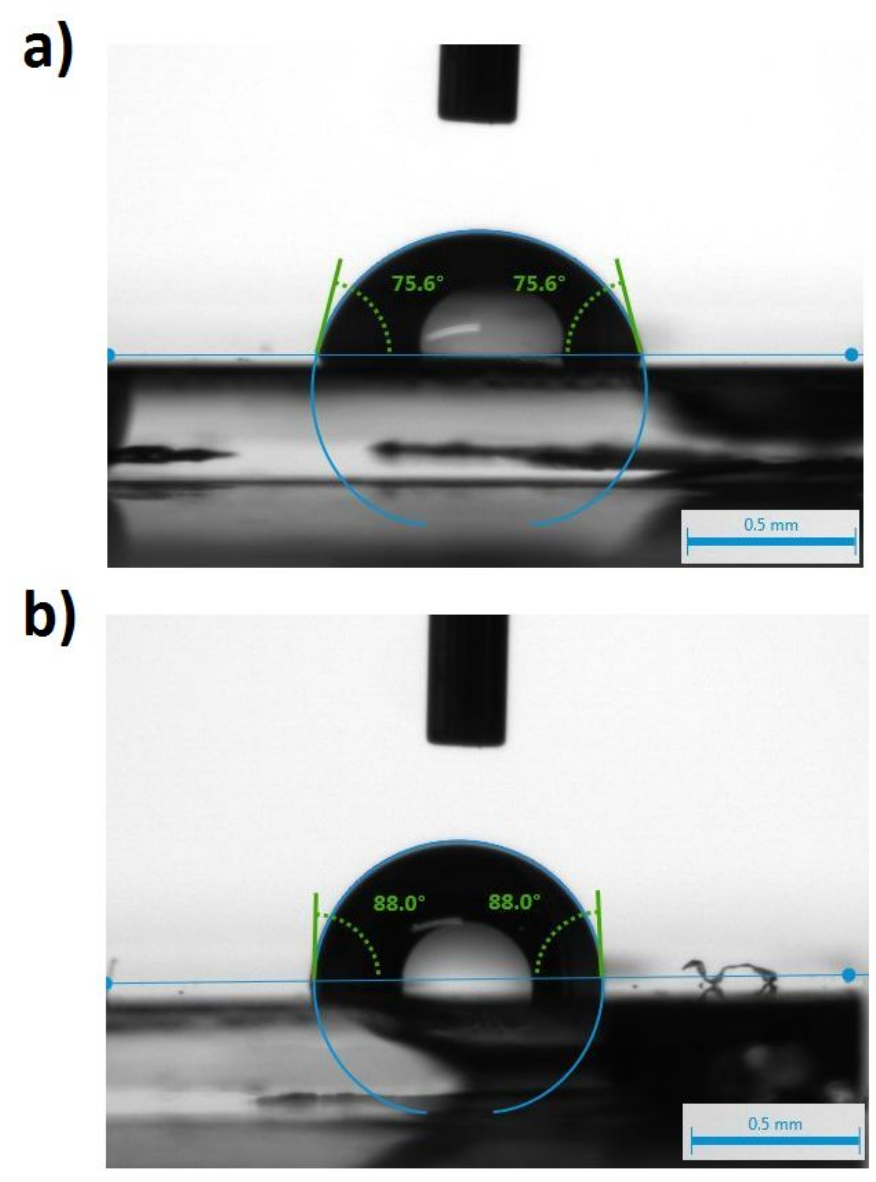

Figure S7. Water contact angles of: a) glass $/ \mathrm{MAPbI}_{3}$ and b) glass $/ \mathrm{MAPbI}_{3} / \mathrm{CMB}-\mathrm{vTA}$.

\section{References}

1. Shalom, M.; Guttentag, M.; Fettkenhauer, C.; Inal, S.; Neher, D.; Llobet, A.; Antonietti, M. In Situ Formation of Heterojunctions in Modified Graphitic Carbon Nitride: Synthesis and Noble Metal Free Photocatalysis. Chem. Mater. 2014, 26 (19), 5812-5818.

2. Kumru, B.; Cruz, D.; Heil, T.; Schmidt, B. V. K. J.; Antonietti, M. Electrostatic Stabilization of Carbon Nitride Colloids in Organic Solvents Enables Stable Dispersions and Transparent Homogeneous CNFilms for Optoelectronics. J. Am. Chem. Soc. 2018, 140, jacs.8b08956. 